\title{
Influence des états de surface du territoire agricole sur le déclenchement des inondations catastrophiques
}

\author{
F Papy ${ }^{1,}$ C Douyer 2 \\ 1 INRA-SAD, 78850 Thiverval-Grignon; \\ 2 SRAE, 1 rue Dufay, 76100 Rouen, France
}

(Reçu le 8 octobre 1990; accepté le 20 janvier 1991)

\begin{abstract}
Résumé - Dans les régions de grande culture du Nord-Ouest de l'Europe, certaines situations morphologiques et pédologiques bien identifiées peuvent générer des inondations catastrophiques par émission et concentration de ruissellement. L'état de surface du territoire agricole influe-t-il sur la manifestation de ces phénomènes exceptionnels de la même manière qu'il le fait sur ceux qui sont plus fréquents et moins graves ? C'est la question abordée dans ce texte.

L'étude est conduite à partir de l'analyse d'une chronique des catastrophes sur 30 ans dans le pays de Caux (Seine Maritime). Deux types de catastrophes sont distingués : celles qui succèdent à de longues périodes de pluies et surviennent en hiver; celles qui sont dues à des orages et se produisent au printemps.

L'état de surface du territoire agricole a un rôle déterminant sur le déclenchement des inondations; on le constate si I'on examine la répartition des catastrophes au cours de l'année, ainsi que leur chronologie au cours des 30 ans. La maîtrise des inondations doit donc être conçue à partir du territoire agricole situé en amont.
\end{abstract}

inondation / catastrophe / dégradation structurale / limon / ruissellement / érosion par ruissellement concentré / système de culture / bassin versant / pays de Caux

Summary - Relationship between soil surface state of agricultural land and catastrophic flooding. In the arable regions of north-western Europe, some morphological sites can generate runoff and thus cause catastrophic flooding. The paper addresses the following questions: does soil surface state influence these exceptional events as it does for more frequent events? In the Pays de Caux (near the mouth of the Seine), a particularly sensitive region, an inventory of catastrophic flooding, based on events recorded in a local weekly, has been made over a 30-yr period. These events are presented according to their occurrence throughout the year (fig 2), their chronology over the 30-yr period (fig 3) and their spatial repartition (fig 2). Two kinds of catastrophe can be distinguished: 1), winter floods which result from long rainy periods (table III) and need large watersheds (table I); those which cause the most damage are floods due to an exceptionally long and rainy period during wheat sowing; after that time, they are late enough to allow the soil surface to become crusted (table VI); 2), the flash flood caused by spring storms do not need large watersheds (table I). They are correlated both with storm frequency in spring and the amount of winter rain (table IV). However, the correlation of these catastrophes with spring storms is not perfect in that the highest frequency of spring floods appears in June but storms are equally likely to occur in May and in June (fig 4); this is interpreted as an effect of soil surface state. Large amounts of winter rain have a tendency to create rills which in turn increase spring catastrophes by reducing the runoff concentration time. The chronology of flash flood over the $30 \mathrm{yr}$ (fig 3) can be explained by climatic conditions but also by agricultural land use. With agricultural inventories (table II), it is possible to induce soil surface states in January and May (table $V$ ) and thus represent, for a long period, the evolution of soil surface state in these 2 months (fig 5). It would seem that the floodings in the last $10 \mathrm{yr}$ were caused by the increase in streaming surfaces. So the distribution of catastrophes throughout the year as well as over a 30-yr period show the effect of soil surface state of agricultural land on flooding. Thus flood control should be planned taking into consideration the soil surface state of the agricultural area.

flooding / catastrophe / soil surface sealing / loam / runoff / concentrated flow erosion / cropping system / watershed / pays de Caux 


\section{INTRODUCTION}

En dépit de pentes faibles et de conditions climatiques modérées, certaines zones du NordOuest de l'Europe sont le siège de phénomènes érosifs atteignant parfois des dimensions catastrophiques (Pihan, 1979; Maucorps, 1982; De Ploey, 1986; Chisci et Morgan, 1986; Monnier et al, 1986; Auzet, 1987(a); Gabriels et al, 1988). Le phénomène n'est pas nouveau, comme le montrent Hénin et Gobillot (1950), Lefèvre (1958) et des chroniques plus anciennes (Cochet, 1842, 1850; Banse, 1935).

Le système érosif dominant est l'érosion par ruissellement concentré. II se caractérise par une nette dissociation spatiale des zones de collecte du ruissellement (impluvium : versants et tête de vallon) et d'érosion (ligne de concentration : talweg). II se manifeste dans des situations caractérisées par des critères morphologiques, pédologiques et agraires bien particuliers, comme l'ont montré Auzet et al (1990). Sur le rebord des plateaux, les vallons secs constituent des dispositifs morphologiques particulièrement propices à la captation des écoulements superficiels et à leur concentration dans les talwegs; la texture limoneuse des sols de la ceinture limonolœssique du Bassin parisien leur confère une forte aptitude à la battance sous l'action des pluies et, par suite de la baisse de perméabilité qui en résulte, à ruisseler, même à la suite d'épisodes pluvieux de faible intensité; les cultures annuelles, qui occupent une place importante dans ces régions de grande culture et laissent la surface nue une partie de l'année, exposent la surface du sol à l'action dégradante des pluies.

Les situations précédentes donnent lieu à des phénomènes de ruissellement et d'érosion qui peuvent être déclenchés par des événements climatiques de faible intensité et sont, par conséquent, fréquents. Nous qualifieront par la suite ces phénomènes de "chroniques". Dans ces conditions, pour un épisode pluvieux donné, l'état de surface de l'impluvium a un effet décisif sur les quantités ruisselées et les volumes arrachés aux talwegs.

Boffin et al (1988) ont montré, par un suivi de l'évolution des états de surface de parcelles culturales, que le déclenchement de l'érosion en aval n'apparaît qu'à partir d'un certain état de dégradation de la surface du sol en amont. À l'échelle de petits bassins versant élémentaires, des travaux en cours ont établi que la variable ayant le plus fort pouvoir explicatif des volumes érodés en fin d'hiver était la superficie ayant atteint le faciès ultime de la dégradation par le pluies (croûtes de dépôt).

À ce stade des connaissances, une question se pose : pour des événements climatiques exceptionnels suceptibles d'engendrer des catastrophes, l'état de surface du sol conserve-t-il l'importance qu'il a dans les cas plus fréquents, mentionnés ci-dessus ?

On comprend l'acuité de la question quand on sait que les dégâts en dehors du territoire agricole alarment l'opinion et représentent un coût généralement bien supérieur à celui supporté par l'agriculture (Auzet, 1987 (b); Papy et Douyer, 1988; Boardman, 1990; Robinson et Blackman, 1990). Ces dégâts se manifestent par des inondations boueuses traduisant l'effet d'un ruissellement important accompagné d'érosion. Selon la réponse à la question précédente, on pourra plus ou moins compter sur les techniques envisagées pour réduire les risques de dégâts causés par les phénomènes fréquents se développant sur de petits bassins versants (Papy et Boiffin, 1988 (a, b); Ouvry 1989-1990) pour réduire les risques de dégâts catastrophiques d'aval.

Comme les états de surface se modifient au cours de l'année, (Monnier et al, 1986; Papy et Boiffin, 1988 (a); Casenave et Valentin, 1989; Imeson et Kwaad, 1990), nous examinerons si les risques de catastrophes sont liés à leur évolution. Mais les états de surface et leur aptitude au ruissellement dépendent, sur un territoire donné, de la nature de l'occupation agricole (Boardman, 1990); aussi étudierons-nous également la variation des risques de catastrophes entre situations se différenciant par l'assolement.

\section{MÉTHODE}

\section{Principe}

À partir d'un nombre élevé d'événements catastrophiques, nous nous proposons de rechercher les conditions climatiques qui, leur étant communes, paraissent nécessaires au déclenchement du phénomène. Puis, identifiant des situations où, les conditions précédentes étant remplies aucune catastrophe n'a été enregistrée, nous nous interrogerons sur la différence entre les situations avec et sans catastrophes : n'estelle pas liée à l'état de surface du territoire agricole en amont? 
Cependant, l'étude des catastrophes pose des problèmes de méthode liés au caractère de rareté desphénomènes et à la nécessité, pour les étudier, d'avoir recours à des documents historisques. Afin de disposer d'un nombre suffisant d'événéments, comme leur fréquence d'apparition est faible, il faut prospecter une longue série d'années ainsi qu'un vaste espace géographique. Or, plus on élargit la prospection, plus on multiplie le nombre de variables susceptibles d'influencer le déclenchement du phénomène. La diversité des situations ainsi réunies pourrait être mise à profit pour rechercher un modèle d'estimation des risques de catastrophes si elles étaient toutes bien caractérisées de façon homogène par des variables pertinentes. Mais, conduits à reconstituer des événéments passés à travers des documents d'archive, nous ne disposons bien souvent que de données incomplètes et imprécises. Aussi serons-nous amenés à faire des hypothèses que nous expliciterons au cours de la discussion des résultats et ne pourrons-nous pas combiner les données en un dispositif rigoureusement démonstratif d'un modèle explicatif.

\section{Mise en œuvre}

\section{Région d'étude et série climatique}

\section{Le pays de Caux, Seine Maritime}

II constitue une zone tout à fait propice à notre étude. La superficie en plateau proprement dit y est très réduite. De part et d'autre d'une ligne de partage des eaux, la morphologie se présente comme un assemblage de vallons secs orientés les uns vers la Manche, les autres vers la vallée de la Seine (fig 1). Aussi y trouve-t-on, sur une superficie relativement réduite et, de ce fait pour les conditions climatiques voisines, un nombre important de sites exposés à des risques d'inondations catastrophiques dues au ruissellement des terres agricoles. Les terres labourées (TL) occupent $60 \%$ de la surface agricole utile (SAU); le paysage est du type openfield : la circulation du ruissellement d'une parcelle à l'autre ne s'y trouve donc pas entravée et il n'existe pas de haies qui pourraient stocker de l'eau. La couverture limoneuse, dont le taux d'argile croît du nord au sud de 10-15\%, confère au sol au comportement battant. Le modelé général est doux : la très grande majorité des surfaces a une pente comprise entre 1 et $5 \%$; c'est seulement un niveau des raccordements entre vallées sèches et vallées pluviales, que cette dernière peut atteindre $10 \%$.

Une trentaine d'années ont été nécessaires pour avoir une trentaine de journées à catastrophes. Pour les recenser nous avons dépouillé, de 1960 à 1989, l'hebdomadaire local (Le Courrier Cauchois) qui couvre l'ensemble du pays de Caux et dont nous avons vérifié auprès de la direction que le réseau des correspondants locaux est resté stable.

\section{Identification des circonstances catastrophiques}

\section{Circonstances climatiques}

Les renseignements publiés permettent généralement de bien distinguer les inondations provenant des terrains agricoles des simples débordements de rivières ou des remontées de la nappe phréatique; seules les premières ont, bien entendu, été retenues; elles se distinguent des autres par la présence de boue.

Les circonstances climatiques ont été notées dans la presse et complétées par le dépouillement de bulletins météorologiques. On a ainsi identifié si l'épisode pluviométrique, auquel est attribuée la catastrophe, est qualifié ou non d'orageux*. La caractérisation

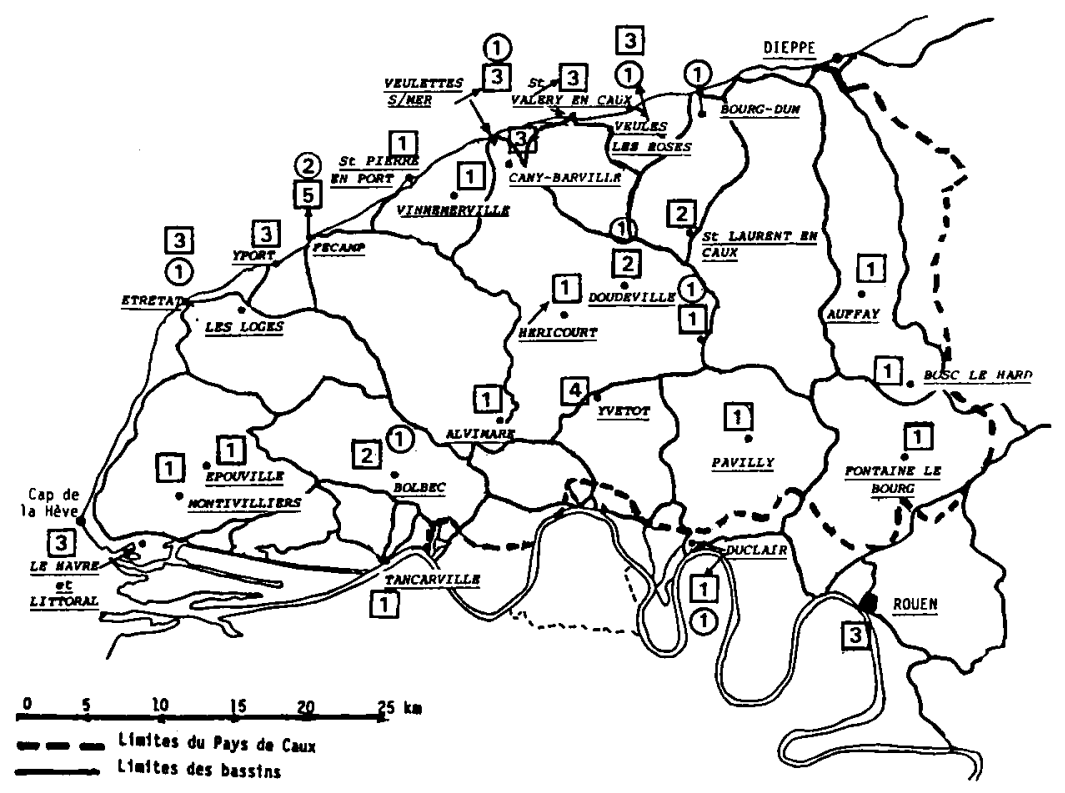

Fig 1. Localisation des catastrophes survenues en 30 ans (de 1960 à 1989) dans le pays de Caux. $\square$ orage; O pas d'orage; à l'intérieur des carrés et des cercles figure le nombre de fois où la localité a été affectée. 
d'une précipitation orageuse en un point donné est délicate : le phénomène est très localisé; sa durée et son intensité ne sont pas connues précisément. Nous avons noté dans le poste météorologique le plus proche :

- l'intensité moyenne pendant la durée de l'orage quand elle est disponible,

- et, dans tous les cas, (orage ou non), la quantité de pluie tombée en $24 \mathrm{~h}$ et dans les 10 et 20 j précédents.

Pendant l'hiver, les types de temps sont dominés par les flux d'ouest et présentent une relative homogénéité à l'échelle régionale. Aussi avons-nous admis que le poste d'Yvetot, au centre de la région, représente bien le territoire étudié. Tandis que, pour évaluer le caractère plus ou moins orageux d'une année, compte tenu de l'aléa de répartition du phénomène dans l'espace, nous avons dénombré les orages identifiés sur l'ensemble des 4 postes où ils sont enregistrés (cap de la Hève, Fécamp, Dieppe et Rouen).

\section{Estimation des surfaces ayant contribué au ruissellement}

Les localités affectées étant identifiées, on peut grâce à la carte des bassins versants du pays de Caux (Camper, 1975) évaluer la superficie qui les domine. C'est une estimation majorée des surfaces susceptibles d'avoir ruisselé. Heureusement les informations contenues dans l'hebdomadaire donnent, dans certains cas, une information plus précise. Sur l'ensemble des 64 catastrophes enregistrées, nous avons pu estimer dans 19 cas les surfaces ayant contribué au ruissellement, aux dires des chroniqueurs (tableau 1). On constate la grande variabilité de ces surfaces.

Tableau I. Superficies reconnues comme ayant entraîné un ruissellement catastrophique $\left(\mathrm{km}^{2}\right)$ dans 19 des cas recensés entre 1960 et 1989.

\section{Type de Surfaces en cause \\ catastrophe}

Sans orages $\quad 16 ; 123 ; 150$

Avec orages $\quad 1 ; 1,5 ; 3 ; 5,8 ; 6 ; 10 ; 11 ; 11 ; 12$

$12 ; 17 ; 25 ; 28 ; 30 ; 90 ; 90$

\section{Évolution des traits du paysage au cours des 30 années}

Certains traits du paysage déterminent l'hydrologie de surface : l'occupation du sol, la présence et la localisation de mares, de bassins de retenue, d'un réseau de chemins et de routes, et de zones urbanisées. La plupart des caractéristiques précédentes ont suivi, au cours du temps, une évolution monotone. L'augmentation continue des surfaces urbanisées, du réseau macadamisé, la diminution des mares, accroissent les risques d'inondation et les dégâts qu'elles produisent. Risques et dégâts sont, au contraire, réduits par les bassins de retenue construits, surtout depuis 1980, en amont de quelques uns des sites les plus exposés.

L'occupation du sol, qui détermine les états de surface et leur évolution au long de l'année, n'a pas suivi d'évolution monotone. Les 4 recensements généraux de l'Agriculture le montrent bien (tableau II). Le rapport STH/SAU passe, entre 1955 et 1970 , de $38 \%$ à $50 \%$, se maintient approximativement à cette valeur au recensement de 1979 , puis diminue pour revenir à $40 \%$ en 1988. Comme les surfaces en prairie n'émettent pratiquement pas de ruissellement, la variation de leur superficie au cours des ans entraîne une variation en sens inverse des risques d'inondations.

Le tableau II donne la répartition des cultures annuelles. Comme le semis représente le dernier stade où le sol se trouve dans un état fragmentaire -donc filtrant-, avant que l'action des pluies n'y provoque la formation de croûtes imperméables, nous avons classé ces cultures en fonction des périodes d'implantation**. C'est ainsi que nous avons distingué les cultures semées en automne, de celles qui le sont au début du printemps (fin février-début mars à fin marsdébut avril) et des suivantes.

Le trait le plus saillant de l'évolution des cultures est le transfert des semis de début printemps vers l'automne, l'avoine puis l'orge de printemps étant progressivement, de 1970 à 1988, remplacées par des orges d'hiver et des escourgeons, glissement que ne compense que partiellement et tardivement l'arrivée du pois.

\section{RÉSULTATS ET DISCUSSION}

\section{Identification des conditions climatiques nécessaires au déclenchement des catastrophes}

\section{Existence de deux types de catastrophes}

Sur les 30 ans, 29 journées à catastrophe ont été dénombrées, et 64 cas de catastrophes, si

\footnotetext{
- Les orages sont des perturbations locales, brusques, de faible durée, qui se manifestent par de fortes averses accompagnées de phénomènes électriques (éclairs, coups de foudre) et souvent de rafales de vent.

** Nous avons vérifié qu'en 1960 les désherbants chimiques sur céréales étaient déjà bien généralisés, faisant ainsi disparaître les hersages pratiqués auparavant; seule reste travaillée en cours de culture une partie de la sole de betterave, ce qui ne représente qu'une faible surface.
} 
Tableau II. Evolution de l'occupation du sol dans le pays de Caux depuis 1955 à partir des Recensements généraux de l'agriculture (source : Service statistique de la direction de l'Agriculture et des Forêts de Seine-Maritime).*

\begin{tabular}{|c|c|c|c|c|}
\hline Année & 1955 & 1970 & 1979 & 1988 \\
\hline $\begin{array}{l}\text { Surface agricole utile } \\
\text { (SAU) (ha) } \\
\text { Terres labourables } \\
\text { (TL) (ha) } \\
\text { Surface toujours en herbe } \\
\text { (STH) (ha) } \\
\text { STH/SAU (\%) } \\
\text { Cultures annuelles (CA) (ha) }\end{array}$ & $\begin{array}{c}231000 \\
139806 \\
89958 \\
38,9 \\
126806\end{array}$ & $\begin{array}{c}228413 \\
112616 \\
114561 \\
50,2 \\
99891\end{array}$ & $\begin{array}{c}220872 \\
116147 \\
103776 \\
47,0 \\
110701\end{array}$ & $\begin{array}{c}218158 \\
133924 \\
82800 \\
38,0 \\
129619\end{array}$ \\
\hline $\begin{array}{l}\text { Semis d'automne } \\
\text { Blé/CA }(\%) \\
\text { Cér second/CA (\%) } \\
\text { Colza/CA }(\%) \\
\text { Total }\end{array}$ & $\begin{array}{c}33,8 \\
1,8 \\
0 \\
35,6\end{array}$ & $\begin{array}{r}29,3 \\
0,6 \\
0,6 \\
30,5\end{array}$ & $\begin{array}{r}32,0 \\
7,1 \\
1,8 \\
40,9\end{array}$ & $\begin{array}{r}32,5 \\
13,5 \\
5,1 \\
51,1\end{array}$ \\
\hline $\begin{array}{l}\text { Semis précoce de printemps } \\
\text { Cér second/CA (\%) } \\
\text { Pois/CA }(\%) \\
\text { Colza/CA }(\%) \\
\text { Lin/CA }(\%) \\
\text { Total }\end{array}$ & $\begin{array}{c}19,1 \\
0 \\
1,3 \\
10,4 \\
30,8\end{array}$ & $\begin{array}{c}24,4 \\
0 \\
1,3 \\
7,2 \\
32,9\end{array}$ & $\begin{array}{c}16,5 \\
0,7 \\
0 \\
7,8 \\
25,0\end{array}$ & $\begin{array}{c}2,0 \\
9,1 \\
0 \\
9,3 \\
20,4\end{array}$ \\
\hline $\begin{array}{l}\text { Semis tardifs de printemps } \\
\text { Betterave (BS + BF)/CA (\%) } \\
\text { Pomme de terre/CA (\%) } \\
\text { Maïs (MG + MF)/CA (\%) } \\
\text { Divers (CF surtout)/CA (\%) } \\
\text { Total }\end{array}$ & $\begin{array}{c}17,3 \\
2,3 \\
? \\
? \\
33,6\end{array}$ & $\begin{array}{r}19,4 \\
2,7 \\
3,8 \\
10,7 \\
36,6\end{array}$ & $\begin{array}{r}18,3 \\
2,7 \\
7,7 \\
5,4 \\
34,1\end{array}$ & $\begin{array}{r}12,5 \\
2,5 \\
12,3 \\
1,2 \\
28,5\end{array}$ \\
\hline
\end{tabular}

\footnotetext{
* Le recensement général agricole (RGA) ne fait pas la distinction entre céréales (et colza) d'hiver et de printemps. Aussi avonsnous utilisé les annuaires des statistiques agricoles qui paraissent annuellement par département pour connaître la proportion relative des surfaces semées aux deux périodes. Cette dernière a été appliquée aux valeurs totales des superficies de céréales et colza du pays de Caux. BS: betterave sucrière; BF: betterave fourragère; MG: maïs grain; CF: cultures fourragères; CA: cultures annuelles; TL: terres labourables; STH : prairies temporaires et artificielles.
}

l'on tient compte qu'une même journée peut en connaître plusieurs dans des localités différentes. S'y ajoutent, en 1966, 2 catastrophes qui ont affecté l'ensemble du pays de Caux.

Le recensement des catastrophes permet de les ventiler en fonction de leur fréquence d'apparition au cours des mois de l'année (fig 2), d'en présenter la chronologie sur 30 ans (fig 3 ) et la répartition spatiale (fig 1 ).

De l'examen des conditions climatiques, il résulte qu'on distingue 2 types de catastrophes: celles qui succèdent à de longues séquences pluvieuses (7 sur 29) s'étalent d'octobre à février, avec une exception en août (fig 2); celles qui suivent un orage (22 journées sur un total de 29) ont lieu de mai à septembre avec un net maximum en juin ( 9 sur 22, fig 2).
La localisation à grande écheile des sites où se sont produites les catastrophes met en évidence une relation univoque entre le type de phénomène et la situation topographique. Sur l'ensemble des phénomènes que nous avons enregistrés, nous n'avons identifié de catastrophe sans orage que dans des sites dominés par de grands impluviums; la surface minimale ayant contribué à un événement de ce type est de 1600 ha (tableau I). En revanche, les catastrophes à orage peuvent affecter les localités se trouvant dans les situations topographiques les plus diverses : exutoire de grand bassin-versant aussi bien que proximité d'une ligne de partage des eaux. Les impluviums mis en cause dans ce type de catastrophe peuvent avoir des tailles très différentes, même très petite (de l'ordre de 100 ha) (tableau I). 


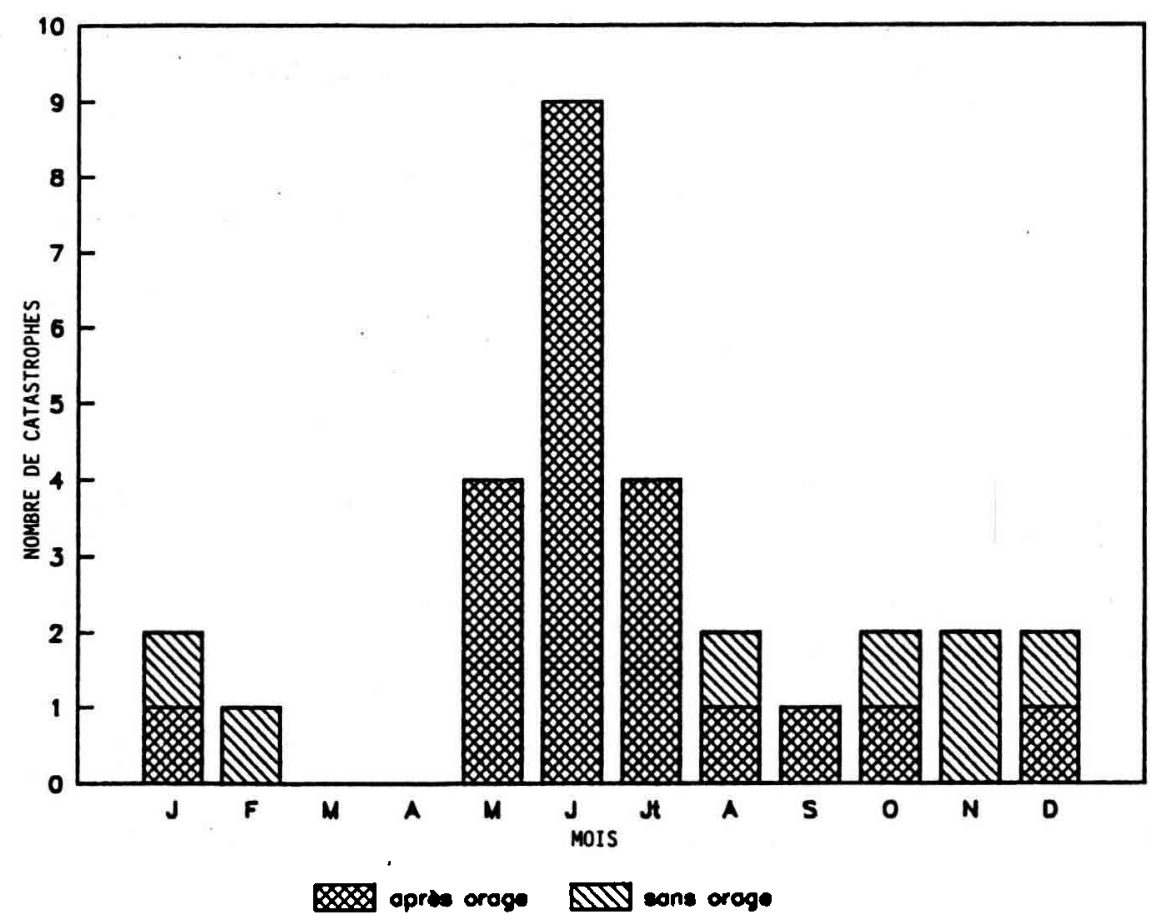

Fig 2. Répartition, au cours de l'année des catastrophes recensées sur 30 ans (de 1960 à 1989) en pays de Caux.

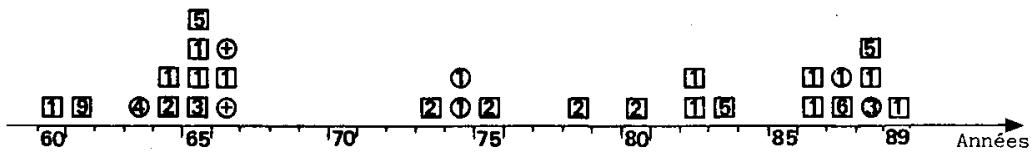

Fig 3. Chronologie sur 30 ans (de 1960 à 1989) des catastrophes recensées dans le pays de Caux; $\square$ catastrophe survenant à la suite d'un orage; O catastrophe ne succédant pas à un orage. À l'intérieur des carrés et des cercles, figure le nombre de localités ayant été affectées. La croix indique que les catastrophes ont affecté l'ensemble du pays.

\section{Conditions pluviométriques des catastrophes sans orage}

Ce type d'événement n'est manifestement pas dû au caractère intensif des précipitations, mais aux quantités d'eau tombées. Les 6 catastrophes d'hiver sont mises en relation avec la pluviométrie du poste d'Yvetot et celle d'été avec celle du poste local. Au tableau IIIA, nous avons reporté la pluviométrie du jour de la catastrophe ainsi que celle des 10 et 20 jours précédents. On en tire un ensemble de conditions "C" $\mathrm{c}$, communes à ces événements :

- pluie du jour de la catastrophe > $15 \mathrm{~mm}$;

- pluie de la décade précédant la catastrophe > $90 \mathrm{~mm}$.

Cependant, sur l'ensemble des 30 années, on recense des séquences pluviométriques correspondant aux conditions «C» qui n'ont pas pour autant entraîné de catastrophes (tableau IIIB).
Ces conditions sont donc nécessaires, mais pas suffisantes. Au total (tableau III dans son ensemble), 20 séquences ont été dénombrées, dont 18 en automne jusqu'à fin janvier et 2 en juillet et août. De ces dernières, l'une a entraîné une catastrophe, l'autre pas. La discussion, qui sera faite plus loin, portera uniquement sur les 18 séquences automnales.

\section{Conditions pluviométriques des catastrophes avec orage}

Sur les 30 ans, 22 journées ont donné lieu à une ou plusieurs inondations catastrophiques dont on a clairement identifié qu'elles ont été déclenchées par un orage. Dix-sept de ces journées se situent en mai, juin et juillet. Mais tous les orages, tant s'en faut, ne donnent pas lieu à catastrophe. 
Tableau III. Les longues séquences pluvieuses de 1960 à 1989 (Poste météo d'Yvetot). * avec éventuellement indice (1) et $(2)=2$ séquences dans une même année. ** Le jour de la catastrophe est inclus dans le total pluviométrique.

\begin{tabular}{lcc}
\hline Année* de & Décade précédant \\
la catastrophe & $\begin{array}{r}\text { Pluie }(\mathrm{mm})^{* *} \\
\text { des } 10 \text { dernierj } j\end{array}$ & des 20 derniers $j$ \\
\hline
\end{tabular}

Séquence pluvieuse ayant provoqué une catastrophe

Séquences automnales

\begin{tabular}{|c|c|c|c|c|}
\hline $1974(1)$ & 02-11 octobre & 34 & 122 & 212 \\
\hline 1963 & $11-20$ novembre & 27 & 95 & 123 \\
\hline $1974(2)$ & 14-23 novembre & 20 & 90 & 144 \\
\hline $1966(2)$ & 03-12 décembre & 17 & 100 & 160 \\
\hline $1965(2)$ & 23 déc-01 janv & 20 & 109 & 177 \\
\hline $1987-1988$ & 23-31 janvier & 30 & 103 & 153 \\
\hline \multicolumn{5}{|c|}{ Séquence non automnale } \\
\hline 1987 & 16-25 août & 70 & 90 & 105 \\
\hline
\end{tabular}

Séquences n'ayant pas provoqué de catastrophe, bien que remplissant les conditions "C»

Séquences automnales

$1982 \quad 02-11$ octobre $\quad 40 \quad 110 \quad 155$

$\begin{array}{llll}1964 & 06-15 \text { octobre } & 39 & 200\end{array}$

$\begin{array}{llll}1960 & 21-30 \text { octobre } & 20 & 117\end{array}$

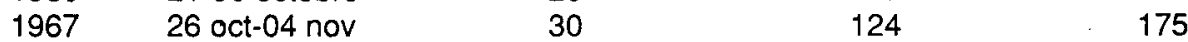

$1966(1) \quad 31$ oct-09 nov $\quad 20 \quad 110 \quad 155$

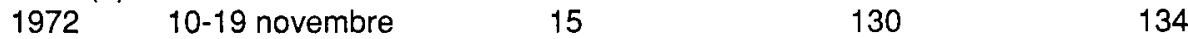

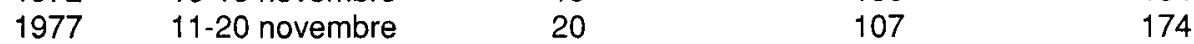

198826 nov-05 déc $\quad 16 \quad 95 \quad 122$

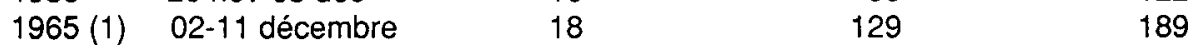

$1981 \quad 07-16$ décembre $\quad 20 \quad 114 \quad 159$

$1979 \quad 09-18$ décembre $\quad 20 \quad 123 \quad 128$

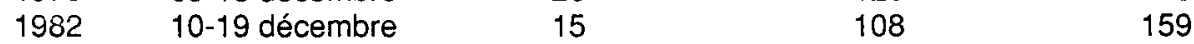

Séquence non automnale

$\begin{array}{llll}1968 & 04-13 \text { juillet } & 15 & 110\end{array}$

Nous ne pouvons caractériser ici les conditions climatiques qui entraînent le phénomène, comme nous l'avons fait dans le cas précédent. Un orage, en effet, est un phénomène mal enregistré dont on ne connaît bien ni la durée, ni l'intensité, ni la position exacte du lieu où le phénomène a été le plus intense. Les orages, par contre, sont beaucoup plus fréquents que les cir- constances climatiques, désignées plus haut par la lettre "C». Aussi est-ce par voie statistique que nous aborderons le problème.

Dans 22 cas les intensités pluviométriques d'orages catastrophiques ont été relevées :

- la moitié des valeurs se situent entre 10 et 35 $\mathrm{mm} / \mathrm{h}$;

Tableau IV. Occurrence des catastrophes de printemps en fonction des pluies hivernales (P) et du nombre d'orages au printemps $(N)$. P: pluies cumulées d'octobre à février; $N$ : nombre d'orages sur 4 postes en mai, juin, juillet; $x / y$ : nombre de journées à catastrophe/nombre d'années correspondant aux caractéristiques de la case.

\begin{tabular}{|c|c|c|c|}
\hline & \multicolumn{2}{|c|}{ Caractère orageux du printemps } & $\begin{array}{l}\text { Somme } \\
\text { des cas }\end{array}$ \\
\hline \multicolumn{4}{|c|}{$\begin{array}{l}\text { Caractère pluvieux } \\
\text { de l'hiver }\end{array}$} \\
\hline $\begin{array}{l}P<480 \\
480<P<500 \\
P>500\end{array}$ & $\begin{array}{l}3 / 6 \\
1 / 2 \\
3 / 7\end{array}$ & $\begin{array}{l}2 / 6 \\
0 / 3 \\
8 / 6\end{array}$ & $\begin{array}{l}05 / 12 \\
01 / 05 \\
11 / 13\end{array}$ \\
\hline Somme des cas & $7 / 15$ & $10 / 15$ & $17 / 30$ \\
\hline
\end{tabular}


- un quart entre 35 et $50 \mathrm{~mm} / \mathrm{h}$;

- un quart sont très élevées (> $70 \mathrm{~mm} / \mathrm{h}$ );

- et sur l'ensemble, 64\% sont supérieures à 26 $\mathrm{mm} / \mathrm{h}$ (pluie d' $1 \mathrm{~h}$ de fréquence décennale relevée à Rouen de 1957 à 1982).

Nous avons établi (tableau IV) pour les mois les plus orageux de mai, juin et juillet la liaison entre le caractère orageux et la fréquence d'apparition des catastrophes. Sur l'ensemble de la série climatique de 30 ans, pour ces 3 mois, le total des orages enregistrés dans les 4 postes présente une valeur médiane de 19. Sur les 15 années les moins orageuses, on enregistre 7 journées à catastrophe contre 10 pour les 15 autres. La liaison statistique n'est donc pas forte.

Les catastrophes provoquées par des orages sont indépendantes des circonstances pluviométriques immédiatement antérieures; elles peuvent survenir, en effet, après des décades sans pluie, comme après une pluviométrie de $90 \mathrm{~mm}$ en $10 \mathrm{j}$. Apparaît par contre une bonne liaison entre les catastrophes de mai, juin, juillet et la pluviométrie hivernale. La mesure de cette dernière la mieux liée au phénomène étudié est le cumul des précipitations d'octobre à février. Sa valeur médiane se situe entre 480 et $500 \mathrm{~mm}$. Sur les 12 années aux hivers les moins pluvieux, on n'enregistre que 5 journées à catastrophes de printemps contre 11 sur les 13 années les plus arrosées en hiver (tableau IV).

Le tableau IV montre que les 2 déterminants étudiés (le caractère orageux du printemps $(N)$ et le caractère pluvieux de l'hiver $(P)$ ) sont indépendants, puisque la valeur de $Y$, correspondant au nombre d'années qui présentent les mêmes caractéristiques, est équilibrée entre les différentes cases du tableau. Ce tableau fait également ressortir l'effet combiné des pluies d'hiver et des orages de printemps sur la probabilité de voie des catastrophes en mai, juin et juillet. Elle est très forte quand la pluviométrie de l'hiver précédent est supérieure à $500 \mathrm{~mm}$ et que le printemps est orageux (plus de 19 otages en 3 mois sur les 4 postes). Sur les 6 années présentant ce caractère, on a compté 8 journées à catastrophe.

\section{Effets de la variation des états de surface en cours d'année}

La répartition des catastrophes au cours de l'année (fig 1) dépend-elle de la variation des états de surface du territoire agricole ? Nous allons déduire ces derniers des proportions respectives des cultures annuelles (tableau II), compte tenu de la règle de succession suivante : les céréales secondaires (avoine, orge, escourgeon) succèdent généralement à un blé.

\section{La saison d'automne et d'hiver}

À l'automne, juste après les semis de céréales, 3 catégories d'occupation du sol se distinguent (tableau V) :

- les surfaces semées en céréales d'hiver : elles couvrent, selon l'année, de 30 à $50 \%$ des cultures annuelles (CA);

- les intercultures avec chaumes; elles correspondent à la somme de toutes les céréales à paille diminuée de la sole d'escourgeon, semée derrière blé : soit, selon l'année de 34 à $54 \%$ des $\mathrm{CA}$;

- les intercultures sans débris végétaux; leur superficie est le complément des précédentes; elle varie entre 10 et $16 \%$ des CA.

Tableau V. Les catégories d'occupation du sol début janvier et début mai (en \% de la surface en cultures annuelles) au cours des 4 recensements généraux agricoles.

\begin{tabular}{|c|c|c|c|c|}
\hline $\begin{array}{c}\text { Catégorie } \\
\text { d'occupation du sol }\end{array}$ & \multicolumn{4}{|c|}{ Années recensements agricoles } \\
\hline \multicolumn{5}{|l|}{ Début janvier } \\
\hline Semis & 35,6 & 30,5 & 40,9 & 51,1 \\
\hline Interculture à chaume & 52,9 & 53,7 & 48,5 & 34,5 \\
\hline Interculture sans chaume & 11,5 & 15,8 & 10,6 & 14,4 \\
\hline \multicolumn{5}{|l|}{ Début mai } \\
\hline Semis d'automne & 35,6 & 30,5 & 40,9 & 51,1 \\
\hline Semis précoce de printemps & 30,8 & 32,9 & 25,0 & 20,4 \\
\hline Semis tardif de printemps & 33,6 & 36,6 & 34,1 & 28,5 \\
\hline
\end{tabular}


Chacune de ces catégories a une aptitude au ruissellement qui lui est propre (Boiffin et al, 1986; Papy et Boiffin, 1988a; Ouvry, 1989-1990). Les intercultures restent en l'état jusqu'aux prochains travaux du sol qui, sur ces sols limoneux, ne débutent guère avant mi-janvier. Derrière les céréales à paille, les opérations de déchaumage qui ont lieu après récolte confèrent au sol une rugosité qui, en permettant une détention de l'eau en surface (Onstad, 1984), rend improbable tout ruissellement lorsque l'intensité pluviale reste modérée. Les autres intercultures, après pois, lin, betterave, pomme de terre, maïs, correspondent à des états de surface plans, lisses, peu encombrés de débris, marqués des traces de roues des chantiers de récolte qui occupent jusqu'à $80 \%$ de la surface. Leur infiltrabilité, de l'ordre de quelques $\mathrm{mm} / \mathrm{h}$ (Martin, 1979) les rend propices à l'émission de ruissellement. Les semis de céréales (et, pour une faible part, de colza) ont une aptitude au ruissellement qui varie de façon très sensible au cours de la saison au fur et à mesure que l'état de surface passe, sous l'action des pluies, du faciès fragmentaire après semis à celui de croûte de dépôt. Cette évolution, au cours de laquelle l'infiltrabilité passe de 30-50 $\mathrm{mm} / \mathrm{h}$ à $1-2 \mathrm{~mm} / \mathrm{h}$ (Boiffin et Monnier, 1986; Ouvry, 1989-1990), peut être modélisée, pour les sols battants comme ceux du pays de Caux, en fonction de la somme des pluies reçues et des interruptions par des séquences de dessiccation (Boiffin, 1984; Boiffin et al, 1988; Eimberck, 1989-1990).

En définitive, au cours de l'automne, c'est essentiellement sur les surfaces semées en céréales d'hiver que se joue la variation de l'aptitude au ruissellement du territoire agricole. Deux éléments antagonistes interfèrent:

- les travaux de préparation des terres accroissent l'infiltrabilité; nous allons admettre en première approximation que les semis s'échelonnent du 1 er octobre au 20 novembre progressant linéairement entre ces 2 dates;

- à partir du semis, l'action des pluies fait évoluer l'état de surface vers le faciès ultime de croûtes de dépôt $\left(F_{2}\right.$ correspondant à une très faible perméabilité; nous allons convenir de simuler l'apparition de ce faciès comme suit : il est atteint pour un cumul des pluies après semis de $60 \mathrm{~mm}$ sans interruption, ou de $90 \mathrm{~mm}$ si la séquence est coupée par $3 \mathrm{j}$ sans pluie (Papy et Boiffin, 1988a). Ces 2 modèles d'évolution, très conventionnels, nous permettent de construire le tableau VI.

Pour chacune des 18 longues séquences pluviales identifiées à l'automne, remplissant les conditions $C$ ( $C f$ tableau III), nous avons estimé les dates d'apparition du faciès $F_{2}$ correspondant aux 5 dates de semis : 10,20,31 octobre, 10 et 20 novembre. Chacune de ces dates $\left(d_{1}-\right.$ $d_{5}$ ) est comparée à celle du début de la séquence pluvieuses (D). Le tableau VI permet d'analyser la différence entre les situations ayant entraîné des catastrophes et les autres. Nous allons le faire en distinguant les cas, selon la position de la séquence pluvieuse, par rapport à la campagne de semis.

Trois séquences ont lieu au tout début des semis de céréales (références 1974(1), 1982, 1964). À cette période, peu de surfaces sont généralement aptes à ruisseler. Celles qui le sont le plus sont les cultures de printemps déjà récoltées (pomme de terre, pois, lin); elles sont, en superficie, moins importantes sur l'ensemble du pays de Caux que les betteraves et maïs non encore récoltées (tableau II), sur lesquelles la fissuration intervenue au cours de l'été redonne à la surface du sol une forte perméabilité, même si elle avait atteint le faciès $F_{2}$. Or, sur les 3 séquences, celle qui débute le 2 octobre 1974 a produit une inondation. On peut penser que l'exceptionnelle pluviométrie de septembre (170 $\mathrm{mm}$, correspondant à une fréquence d'apparition de $1 / 10$ sur la série étudiée) a refermé les surfaces fissurées et fait ruisseler l'ensemble des surfaces en cultures de printemps, récoltées ou non, soit un pourcentage des CA qui se situe entre 69 et $59 \%$, selon les données du tableau II.

En pleine saison de semis, 2 séquences de conditions «C $\mathrm{C}$. donnent une catastrophe, 5 n'en donnent pas. Après la date du 20 novembre, choisie conventionnellement comme fin des semis, 3 séquences sont désastreuses contre 5 .

Si l'on examine l'ensemble de ces données, on constate qu'il n'y a pas de catastrophe lorsque $d_{1}$ est postérieur à $D(7$ cas repérés sur le tableau VI par les dates "d" en caractères gras); un cas fait exception : 1963, où l'inondation très localisée doit avoir des causes conjoncturelles que nous n'avons pas les moyens de donner. Dans les autres cas où $d_{2}$ au moins est antérieur à $D$, les catastrophes se produisent :

- soit à la suite d'une campagne de semis peu pluvieuse au début (1965(2) et 1987-1988, répérable par une durée importante entre les premières dates de semis et les dates $d_{1}$ et $d_{2}$ leur correspondant; dans ce cas, toute la sole de blé a atteint le faciès $F_{2}$ et il est tombé au moins $100 \mathrm{~mm}$ avant la date $\mathrm{D}$; 


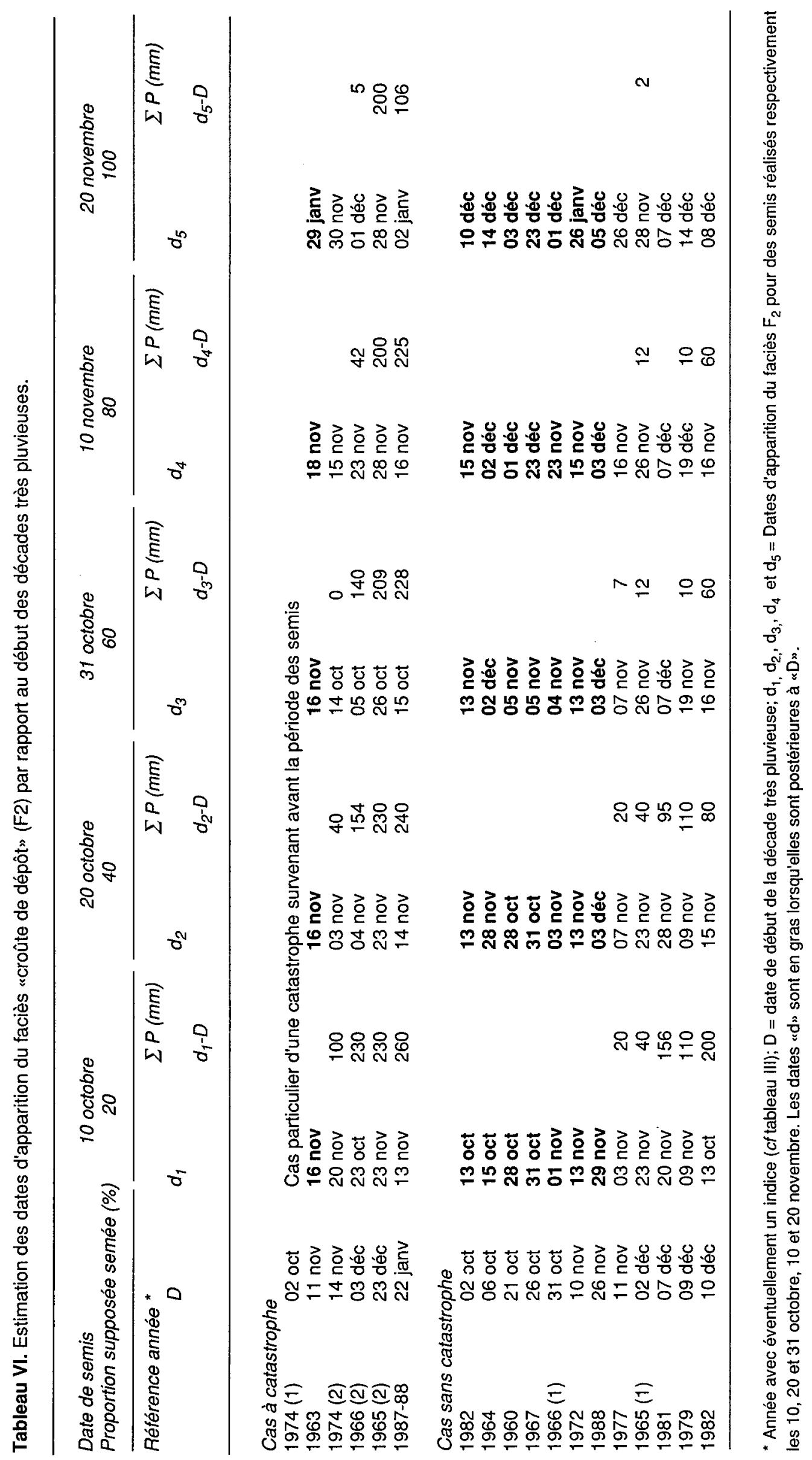


- soit, à la suite d'un automne pluvieux dès le début (1974(2), 1966(2)), au cours duquel les récoltes tardives de maïs et betterave se font en mauvaises conditions et constituent des surfaces facilement ruisselantes; dans ce cas, il peut y avoir catastrophe, même avant la fin des semis de blé (1974(2)).

Ainsi comprend-on pourquoi les conditions «C» ne sont pas suffisantes pour déclencher des catastrophes; il faut de plus, que l'état du territoire agricole ait atteint, sur une superficie suffisante, un état poussé de dégradation de la structure en surface. Mais, alors que dans les phénomènes chroniques tels qu'ils ont été étudiés par Boiffin et al (1988), des symptômes d'érosion se manifestent dès l'apparition du faciès $F_{2}$ en aval, il faut une quantité supplémentaire de pluie -au moins $100 \mathrm{~mm}$ d'après nos données- pour déclencher une inondation. On le constate bien en comparant 1965(1) à 1965(2) et 1987-1988 (tableau VI). Cette quantité de pluie supplémentaire correspondrait, en moyenne, à la capacité de stockage des grandes surfaces nécessaires au déclenchement des inondations hivernales. En suivant en temps réel le déclenchement d'une inondation, Ouvry (1987) évalue une capacité de stockage tout à fait comparable à celle que nous proposons.

\section{La saison de printemps et d'été}

Au printemps, une fois achevés les derniers semis, 3 catégories de surfaces peuvent être distinguées au sein des cultures annuelles (tableau V) :

- les semis d'automne ( $30-50 \%$ des CA) : ils présentent une forte et constante aptitude à ruisseler;

- les semis réalisés précocement au printemps (20 à $33 \%$ des CA); il s'agit des céréales de printemps, du pois et du lin; leur aptitude à ruisseler augmente au fur et à mesure que se dégrade, sous l'effet des pluies, la structure de surface;

- les semis tardifs de printemps (28 à $36 \%$ des $\mathrm{CA})$, qui correspondent aux betteraves, pommes de terre, maiis et cultures fourragères diverses; des 3 catégories ce sont, au mois de mai, les moins aptes à ruisseler.

La figure 4 met en regard le nombre d'orages mensuels pendant la période étudiée sur l'ensemble des 4 postes retenus et le nombre de journées ayant connu une ou plusieurs catastrophes. On constate que si chacun des 3 mois (mai, juin et juillet) connaît autant d'orages, c'est

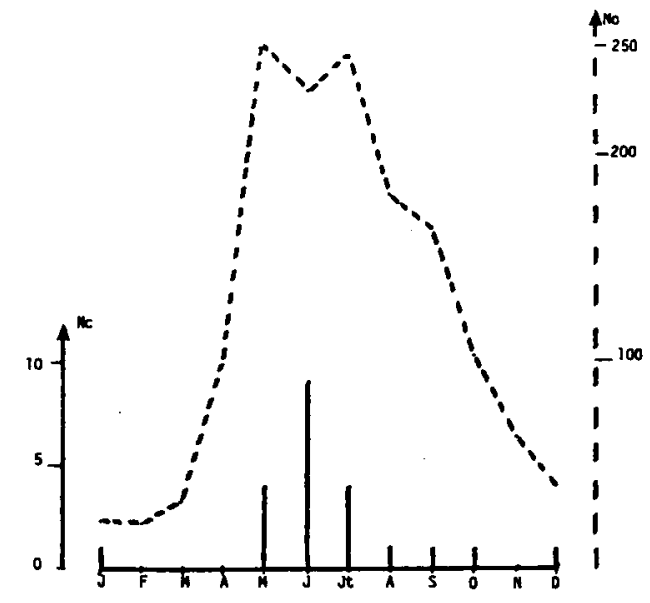

Fig 4. Répartition mensuelle des orages (No) et des catastrophes à orages (Nc) en pays de Caux; No : Nombre total de jours d'orage par mois sur 4 postes météo; Nc : Nombre de journées orageuses ayant produit une catastrophe sur la série des 30 années.

bien en juin qu'on enregistre le plus de catastrophes. Sous l'hypothèse que statistiquement de mai à juillet les orages présentent les mêmes caractéristiques d'intensité et de durée, ce résultat laisse supposer un effet moyen de la surface du sol. Nous proposons de l'interpréter comme suit : en mai l'état de surface ne serait pas assez dégradé sur une superficie suffisante pour entraîner du ruissellement catastrophique; à cette époque les semis les plus tardifs présenteraient une infiltrabilité encore supérieure aux intensités pluviométriques enregistrées comme ayant pu déclencher des dégâts (voir plus haut); tandis qu'en juillet la dessiccation et la fissuration du sol, agissant sur l'ensemble de la superficie, suffiraient à augmenter à nouveau l'infiltrabilité du sol pour réduire les risques.

La liaison enregistrée entre la pluviométrie d'octobre à février et les catastrophes orageuses de printemps ne peut pas s'expliquer par l'acquisition, pour les impluviums, d'une aptitude à ruisseler. Plus de la moitié des CA est en effet fragmentée par les préparations de semis après le mois de février. Aussi doit-on invoquer un autre processus. Meynier (communication orale) propose l'explication suivante. Après un hiver pluvieux, les figures d'érosion en fond de talweg sont plus nombreuses et plus profondes que d'habitude. Même si, au début du printemps, les façons culturales en effacent certaines, subsistent celles faites dans les céréales d'hiver. En réduisant le temps de concentration du ruissellement grâce au réseau de collecte qu'elles constituent, elles augmenteraient les risques de catastrophes. 
Rappelons ici que dans les conditions orageuses qui déclenchent les catastrophes de printemps de petits impluviums peuvent suffire à produire des débits générateurs de dégâts.

\section{Effet de l'évolution de l'occupation du sol de 1960 à 1989}

Nous allons maintenant étudier la variation des catastrophes au cours des 30 ans (fig 3). L'apparition de ces dernières est-elle liée à l'évolution de l'occupation du sol pendant cette série chronologique?

Les tableaux II et $\mathrm{V}$ permettent de construire la figure 5 illustrant la variation de l'occupation du sol en début janvier et début mai entre 1955 et 1988 . Cette représentation permet de distinguer comme suit 3 séries de 10 ans :

Tableau VII. Aptitude à ruisseler du territoire agricole en fonction des séries d'années.

\begin{tabular}{|c|c|c|}
\hline Série & Année & Aptitude à ruisseler du territoire agricole \\
\hline A & $\begin{array}{l}1980-1989 \\
1960-1964\end{array}$ & Forte \\
\hline B & [ & Moyenne \\
\hline $\mathrm{C}$ & $1965-1974$ & Faible \\
\hline
\end{tabular}

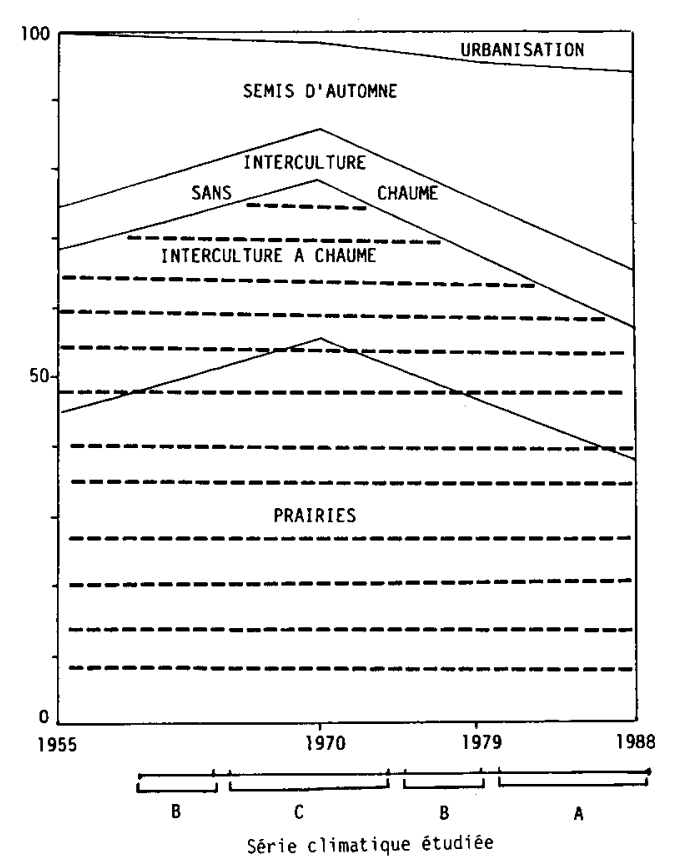

On ne peut discuter d'un effet de l'occupation du territoire agricole sur les catastrophes qu'en examinant comment se distribuent, au sein de ces séries, les conditions climatiques de déclenchement.

Nous avons vu que nous ne pouvions interpréter l'effet des conditions «C" qu'en les resituant par rapport à la campagne des semis. Après le 20 novembre, date supposée de fin des semis, elles ne sont réalisées que 8 fois en 30 ans : 4,1 et 3 fois respectivement dans les séries $A, B$ et C. Cette répartition est trop mauvaise pour permettre une interprétation de l'effet mode d'occupation du territoire sur les catastrophes hivernales.

La réalisation des conditions climatiques favorables aux catastrophes de printemps est donnée au tableau VIII pour chacune des 3 séries d'années. Elle n'y apparaît pas bien répartie; les séries se classent comme suit :

- $C>A>>B$, pour le caractère orageux du printemps,

$-\mathrm{A}>\mathrm{B}>\mathrm{C}$, pour le caractère pluvieux de l'hiver.

Les 2 séries les plus extrêmes sur l'aptitude à ruisseler présentent cependant les conditions climatiques les plus voisines:

-6 printemps orageux pour $A$, contre 7 pour $C$, -5 hivers pluvieux pour $A$, contre 4 pour $C$.

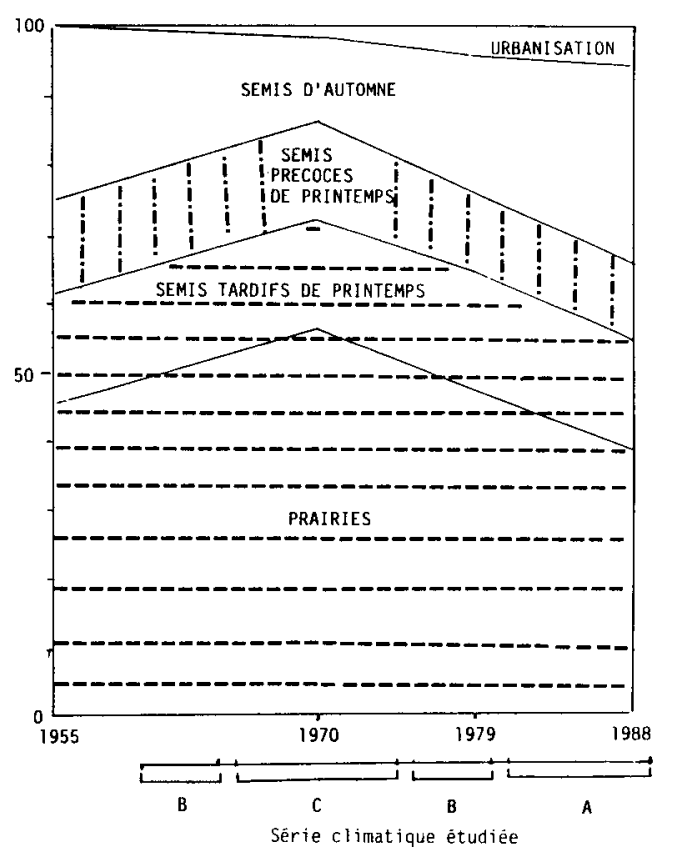

Fig 5. Évolution des états de surface entre 1955 et 1989 sur le territoire du pays de Caux. $100=$ SAU en 1955 (SAU : Surface Agricole Utile).

\begin{tabular}{|ll}
\hline I- Début janvier & II - Début mai \\
\hline I & $\begin{array}{l}\text { Ruissellement } \\
\text { Ruissellement partiel }\end{array}$ \\
Pas de ruissellement & Sous faible intensité pluviométrique
\end{tabular}


Tableau VIII. Occurrence des catastrophes de printemps en fonction de la pluviométrie hivernale $(P)$ et du nombre d'orages $(N)$ pour 3 séries d'années climatiques correspondant à différentes aptitudes du territoire agricole à ruisseler. * $A$ : de 1980 à $1989 ; B:$ de 1960 à 1964 et de 1975 à 1979; $C:$ de 1965 à 1974.

\begin{tabular}{|c|c|c|c|c|c|c|c|c|c|}
\hline & \multicolumn{2}{|c|}{$A$} & \multirow{2}{*}{$\begin{array}{l}\text { Somme } \\
\text { des cas }\end{array}$} & \multicolumn{2}{|c|}{$B$} & \multirow{2}{*}{$\begin{array}{l}\text { Somme } \\
\text { des cas }\end{array}$} & \multicolumn{2}{|c|}{$C$} & \multirow{2}{*}{$\begin{array}{l}\text { Somme } \\
\text { des cas }\end{array}$} \\
\hline & $N<19$ & $N>19$ & & $N<19$ & $N>19$ & & $N<19$ & $N>19$ & \\
\hline$P<480$ & $3 / 2$ & $0 / 1$ & $3 / 3$ & $0 / 3$ & $1 / 1$ & $1 / 4$ & $0 / 1$ & $1 / 4$ & $1 / 5$ \\
\hline $480<P<500$ & & $0 / 2$ & $0 / 2$ & $1 / 2$ & & $1 / 2$ & & $0 / 1$ & $0 / 1$ \\
\hline$P>500$ & $2 / 2$ & $4 / 3$ & $6 / 5$ & $0 / 3$ & $1 / 1$ & $1 / 4$ & $1 / 2$ & $3 / 2$ & $4 / 4$ \\
\hline Somme des cas & $5 / 4$ & $4 / 6$ & $9 / 10$ & $1 / 8$ & $2 / 2$ & $3 / 10$ & $1 / 3$ & $4 / 7$ & $5 / 10$ \\
\hline
\end{tabular}

Or la série A contient plus de journées catastrophiques que $C$ (9 contre 5 ), ce qui met en évidence un effet de l'occupation du sol sur la fréquence des catastrophes de printemps. Un constat renforce cette conclusion : dans la série A, 3 catastrophes surviennent au cours de 2 années pourtant peu propices au déclenchement du phénomène, puisqu'elles sont à la fois peu orageuses et peu pluvieuses en hiver. Dans aucune des autres séries de telles conditions n'ont de conséquences désastreuses.

Nous avons procédé à un test de sensibilité en faisant varier les bornes des séries d'une année de part et d'autre; les conclusions ne s'en trouvent pas modifiées.

Sans qu'on puisse évoquer des conditions climatiques particulières, les risques de catastrophes semblent donc avoir augmenté au cours des dernières années. C'est d'autant plus vraisemblable que la construction récente de bassins de retenue en a évité quelques-unes (Ouvry, communication orale). Nos résultats relient cet accroissement du risque d'inondation à une évolution de l'occupation du territoire agricole se traduisant par une augmentation des surfaces aptes à ruisseler. Bien que nos données ne nous permettent pas de le vérifier, on peut penser que cette évolution améliore la continuité des surfaces susceptibles de ruisseler, ce qui, en diminuant les temps de concentration, accroît encore plus les risques d'inondations. Or, utilisant la théorie de la percolation, Stauffer (1985, cité par Gardner et al, 1989) a montré, dans le cas d'une répartition aléatoire, que dès qu'un type de surface occupe $60 \%$ du paysage, il y a continuité. Des développements théoriques seraient à poursuivre dans ce sens sur le ruissellement pour savoir si cette valeur correspond bien à un accroissement des risques du fait de la continuité des surfaces.

Mais, en même temps que l'occupation agricole du territoire, d'autres caractéristiques agraires ont changé concomitamment. À l'augmentation de taille des parcelles, l'opinion courante attribue une part de la croissance des risques. La bibliographie n'en établit nettement d'effet qu'en pays de bocage, où la suppression des haies diminue la capacité de stockage du bassin versant et accroît la vitesse du ruissellement (Alstrom et Bergman, 1988; Burel et Baudry, 1989). Ces raisons interviennent moins dans un paysage d'openfield. Bien sûr l'allongement des longueurs de pente peut augmenter l'érosion de versant. Mais pour ce qui est de la quantité ruisselée, l'accroissement de taille des parcelles n'a qu'un effet local et occasionnel à l'échelle de quelques dizaines d'ha quand l'aléa de l'attribution des cultures aux parcelles met en continuité des surfaces aptes à ruisseler (Papy et Boiffin, 1988a); à l'échelle des superficies nécessaires au déclenchement des catastrophes ( $>100 \mathrm{ha}$ ) il est moins évident que la taille des parcelles intervienne; une modélisation du ruissellement à cette échelle serait à faire cependant pour le vérifier.

L'évolution des techniques culturales est également invoquée pour expliquer l'augmentation des risques. Mais elles peuvent avoir des effets très contradictoires. Aussi est-ce bien la nature de l'occupation du sol qui paraît être prépondérante dans l'évolution des risques de catastrophes.

\section{CONCLUSION}

L'analyse qui vient d'être faite d'une série chronologique de catastrophes dans une région aux sols limoneux, met en évidence que le risque d'inondation catastrophique est lié aux états de surface du territoire agricole : au sein d'une année culturale il augmente au fur et à mesure que se développe le processus de dégradation structurale; entre années, il croît dans le même 
sens que la proportion du territoire susceptible de ruisseler.

Les pluies de faible intensité, courantes en hiver, qui suffisent à provoquer fréquemment de l'érosion chronique, peuvent provoquer des catastrophes sous certaines conditions, rarement remplies, il est vrai. L'aptitude du territoire agricole à ruisseler en hiver influe non seulement sur l'occurence des inondations hivernales, mais sensibilise le terrain au déclenchement de catastrophes printanières, vraisemblablement par la création d'un réseau de circulation de l'eau.

Ces conclusions plaident pour qu'on s'attache à concevoir, à partir de l'amont, la maîtrise des inondations catastrophiques.

\section{REMERCIEMENTS}

Les connaissances expertes de MM Meynier (chambre d'agriculture) et Ouvry (AREAS) nous ont été très utiles pour rédiger ce texte; $M$ Hancart, Chef du service départemental de statistique agricole, nous a donné informations et conseils. A V Auzet, MM Baudry, Ouvry et Poezen ont relu et précieusement annoté le premier texte. Qu'ils reçoivent tous ici nos sincères remerciements.

\section{RÉFÉRENCES}

Alstrom K, Bergman A (1988) Sediment and nutrient losses by water erosion from arable land in south Sweden. A problem with non point pollution? Vatten 44, 193-204

Auzet AV (1987a) L'érosion des sols cultivés en France sous l'action du ruissellement. Ann Géographie 537, 529-556

Auzet AV (1987b) L'érosion des sols par l'eau dans les régions de grande culture : aspects agronomiques. Minist Environ Minist Agric CEREG-UA 95, CNRS

Auzet AV, Boiffin J, Papy F, Maucorps J, Ouvry JF (1990) An approach to the assessment of erosion forms and erosion risk on agricultural land in the northern Paris bassin, France. In: Soil erosion on agricultural land (J Bordman, IDL Foster, JA Dearing, eds) Wiley and Sons, 383-400

Banse D (1935) Histoires des rues de Fécamp. Banse, Fécamp, $164 \mathrm{p}$

Boardman $J$ (1990) Soil erosion on the South Downs: a review. In: Soil erosion on agricultural land (J Boardman, IDL Foster, JA Dearing, eds) Wiley and Sons, 87-105

Boiffin $J$ (1984) La dégradation structurale des couches superficielles du sol sous l'action des pluies. Thèse INA P-G, $320 p+$ annexes
Boiffin J, Monnier G (1986) Infiltration rate as affected by soil-surface crusting caused by rainfall, 210-217. In: Assessement of soil surface sealing and crusting (Callebaut F, Gabriels $G$, de Boodt $M$, eds) Flanders Research Center for Soil Erosion and Soil Conservation, Ghent, $374 p$

Boiffin J, Papy F, Peyre $Y$ (1986) Systèmes de production, systèmes de culture et risques d'érosion dans le pays de Caux. Rapport INA-PG/INRA/ Minist Agric, $154 p+$ annexes

Boiffin J, Papy F, Eimberck M (1988) Influence des systèmes de culture sur les risques d'érosion par ruissellement concentré. I Analyse des conditions de déclenchement de l'érosion. Agronomie 8, 663673

Burel F, Baudry J (1989) Hedgerow network patterns and process in France. In: Changing landscape : an ecological perspective (Zonneveld JS, Forman RTT, eds) Springer Verlag, New-York, 99-120

Camper JF (1975) Étude typologique des bassins versants de Haute-Normandie. Public SRAE de HauteNormandie, $31 \mathrm{p}+$ annexes + carte

Casenave N, Valentin C (1989) Les états de surfaces de la zone sahélienne; influence sur l'infiltration. ORSTOM, $229 \mathrm{p}$

Chisci G, Morgan RPC (eds) (1986) Soil erosion in the european community. Impact of changing agriculture. Balkema, Rotterdam

Cochet (Abbé) (1842) Les inondations; pélerinages à Fécamp, Yport, Vaucotte et Étretat, après les inondations du 24/9/1842. Périaux, Rouen, $30 \mathrm{p}$

Cochet (Abbé) (1850) Etretat, son passé, son présent, son avenir. Delevoie, Dieppe, $84 \mathrm{p}$

De Ploey J (1986) Bodemerosie in de lage landen. Een Europees milieuproblem. Acco, Leuven

Eimberck M (1989-1990) Facteurs d'érodibilité des sols limoneux : réflexions à partie du cas du pays de Caux. Cah ORSTOM, Sér Pédol vol XXV, 81-94

Gabriels D, Cadron W, De Mey P (1988) Provisional rain erosivity maps of some European communities countries. In: Publication of the European Community CD-NA-10860-EN-C. CEE Bruxelles, 93-118

Gardner RH, O'Neill RV, Turner MG, Dale VH (1989) Quantifying scale - dependent effects of animal movement with simple percolation models. Landscape Ecology, 3 (3/4), 217-227. SPB Acad Publ bv, La Haye

Hénin S, Gobillot Th (1950) L'érosion en France. Bull Tech Inf Minist Agric 6, 431-433

Imeson AC, Kwaad FJP (1990) The response of tilled soils to wetting by rainfall and the dynamic character of soil erodibility. In: Soil erosion on agricultural land. (Boardman J, Foster IDL, Dearing JA, eds) Wiley and Sons, 3-14

Lefèvre $P$ (1958) Quelques phénomènes d'érosion en Picardie. Ann Agron 1, 91-129

Martin L (1979) Accelerated soil erosion from tractor wheelings: a case study in Mid-Bedforshire, England. Colloque sur l'érosion des sols en milieu 
tempéré non méditerranéen. Univ L Pasteur, Strasbourg, INRA, 157-161

Maucorps $J$ (1982) A note of farmland erosion in northern France. In: Soil erosion (abridged Proc workshop on soil erosion and conservation, Florence, october), Report EUR 8427, 50-52

Monnier G, Boiffin J, Papy F (1986) Réflexions sur l'érosion hydrique en conditions climatiques et topographiques modérées : cas des systèmes de grande culture de l'Europe de l'Ouest. Cah ORSTOM, Sér Pédol 12, 123-131

Onstad CA (1984) Depressional storage on tilled soil surfaces. Trans Am Soc Agric Eng 27, 729-732

Ouvry JF (1987) Bilan des travaux, campagne 1986 1987, Rapport AREAS

Ouvry JF (1989-1990) Effet des techniques culturales sur la susceptibilité des terrains à l'érosion par ruissellement concentré : expérience du Pays de Caux. Cah ORSTOM, Sér Pédol vol XXV (1-2), 157-169

Papy F, Boiffin J (1988 a) Influence des sytèmes de culture sur les risques d'érosion par ruissellement concentré. II. Évaluation des possibilités de maîtrise du phénomène dans les exploitations agricoles. Agronomie 8, 29-38

Papy F, Boiffin J (1988 b) The use of farming system for the control of runoff and erosion. In: Proc workshop soil erosion (Auerswald and Schertman, eds) Munich, Soil Technol 1, 29-38

Papy F, Douyer C (1988) Les déterminants des catastrophes liées au ruissellement des terres agricoles en Pays de Caux. INRA-SERDA, $44 p$

Pihan J (1979) Risques climatiques d'érosion des sols en France. In: Seminar on agricultural soil erosion in temperate non-mediterranean climate $(\mathrm{H}$ Vogt, $T$ Vogt, eds) Univ Louis Pasteur, Strasbourg-Colmar, 1978, 13-18

Robinson DA, Blackman JD (1990) Some costs and consequences of soil erosion and flooding around Brighton and Hove, Autumn 1987. In: Soil erosion on agricultural land (J Boardman, IDL Foster, JA Dearing, eds) Wiley and Sons, 369-382 